\title{
Engineered nanostructured virus/ZnO hybrid materials with dedicated functional properties
}

Petia Atanasova Dr. rer. nat.

Senior Scientist, Institute for Materials Science, University of Stuttgart, Stuttgart, Germany

Rudolf C. Hoffmann Dr. rer. nat.

Senior Scientist, Eduard-Zintl-Institut für Anorganische und Physikalische Chemie, Technische Universität Darmstadt, Darmstadt, Germany

Nina Stitz Dr. rer. nat.

Scientist, Institute for Materials Science, University of Stuttgart, Stuttgart, Germany

Shawn Sanctis MSc

PhD student, Eduard-Zintl-Institut für Anorganische und Physikalische Chemie, Technische Universität Darmstadt, Darmstadt, Germany

(Orcid:0000-0001-8594-9749)
Zaklina Burghard Dr. rer. nat.

Senior Scientist, Institute for Materials Science, University of Stuttgart, Stuttgart, Germany (Orcid:0000-0003-3906-8582)

Joachim Bill Dr. rer. nat.

Professor, Institute for Materials Science, University of Stuttgart, Stuttgart, Germany

Jörg J. Schneider Dr. rer. nat.

Professor, Eduard-Zintl-Institut für Anorganische und Physikalische Chemie, Technische Universität Darmstadt, Darmstadt, Germany

(Orcid:0000-0002-8153-9491)

Sabine Eiben Dr. rer. nat.

Senior Scientist, Institute of Biomaterials and Biological Systems, University of Stuttgart, Stuttgart, Germany (corresponding author:

sabine.eiben@bio.uni-stuttgart.de) (Orcid:0000-0001-5498-666X)

Bioinspired mineralization for the production of new functional materials offers mild reaction conditions suitable for integrating biological templates and build hierarchically organized hybrid nanostructures with defined properties. In this respect, the tobacco mosaic virus (TMV) stands out due to its unique structural dimensions. Here, the authors present a novel mineralization pathway for the synthesis of virus-based zinc oxide (ZnO) hybrids with multifunctional properties. Wild-type TMV, two TMV mutants (E50Q and TMV-Cys) and amino-functionalized selfassembled monolayers $\left(\mathrm{NH}_{2}\right.$-SAMs), as a reference, were used as templates. This mineralization approach allows control of the particle size of the inorganic phase. Further, the virus contributes additionally to the texturing of zinc oxide. Field-effect transistors (FETs) built from the hybrid films, obtained at close to ambient conditions, showed reproducible results at optimized conditions and without posttreatment. This significantly reduced the threshold voltage of the $\mathrm{E50Q} /$ zinc oxide $\mathrm{FET}$ compared to that of the $\mathrm{NH}_{2}$-SAMs/zinc oxide FET points to the impact of the organic template on FET performance. Nacre-like virus-based zinc oxide multilayers and corresponding monolithic references were prepared. The mechanical properties, namely, Young's modulus, hardness and fracture toughness, were determined and an improvement in the mechanical performance by genetic modification was observed.

\section{Notation}

a measured crack length within coating

$b \quad$ measured crack length on substrate

c virus concentration

$D_{\mathrm{H}} \quad$ hydrodynamic diameter

$D_{\text {XRD }} \quad$ crystallite size calculated from X-ray diffraction data

E Young's modulus

$E_{\mathrm{c}} \quad$ Young's modulus of coating

$E_{\mathrm{s}} \quad$ Young's modulus of silicon substrate

$H$ hardness

$I_{\mathrm{D}} \quad$ drain current

$I_{\text {on/off }}$ on/off current ratio

$K_{\mathrm{c}} \quad$ fracture toughness of coating

$K_{\mathrm{s}} \quad$ fracture toughness of silicon substrate

$n_{\mathrm{I}} \quad$ imaginary part of the refractive index

$n_{\mathrm{R}} \quad$ real part of the refractive index

$n_{\mathrm{RS}} \quad$ refractive index

V volume

$V_{\mathrm{DS}}$ drain-source voltage

$V_{\mathrm{GS}}$ gate-source voltage

$V_{\text {Th }}$ threshold voltage

$v_{\mathrm{w}} \quad$ withdrawal velocity

$\lambda$ dimensionless factor

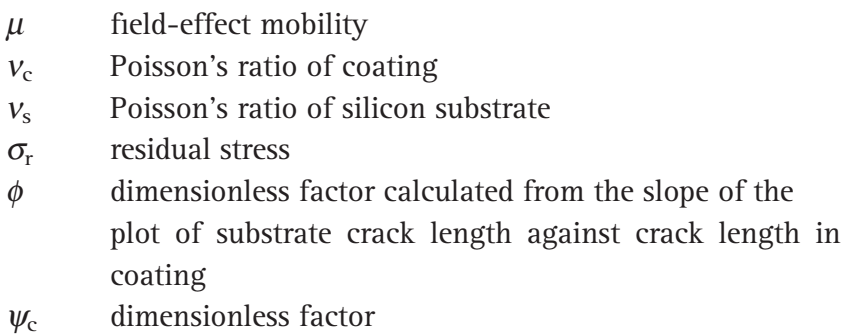

\section{Introduction}

Bioinspired material synthesis provides access to completely novel material classes and combinations which are not (or at least not readily) available by conventional procedures. ${ }^{1}$ Thereby, the interplay between the inorganic part, on the one hand, and the organic component, on the other hand, is of special importance. The organic moiety acts as a structure-directing agent or template; moreover, it is part of the resulting material and thus essential for its properties. Bioinspired material synthesis is increasingly capable of exceeding the mere imitation of structures from nature. The adventure starts when the materials scientist investigates unheard-of combinations to explore materials with novel electronic, piezoelectric or mechanical functions and combinations thereof. $^{2-4}$ 
In continuation of earlier investigations on the chemical bath deposition (CBD) of nanocrystalline zinc oxide $(\mathrm{ZnO})$ on substrates modified with self-assembled monolayers (SAMs), ${ }^{5}$ attempts were made to carry out deposition on tobacco mosaic viruses (TMVs). ${ }^{6}$ This proved to be a very successful and fascinating combination. Under CBD conditions, methanol solution and $60^{\circ} \mathrm{C}$, immobilized TMV is very stable. The shape and length of the virus particle can be altered by adapting the ribonucleic acid (RNA), while the surface properties can be designed by genetic and chemical modification of the coat proteins (CPs) ${ }^{7-11}$ Its stability and unique structure make TMV suitable as a template or scaffold for various applications as diverse as vaccine presentation, chemical sensors and biosensors, battery electrodes, medical imaging and additives in hydrogels. ${ }^{12-17}$ The surface properties of TMV can be modified either genetically (albeit to a small extent) or by biochemical coupling methods using different mutants. ${ }^{18,19}$ Thus, the influence of surface charge and hydrophilicity/hydrophobicity on the assembly of functional materials under different reaction conditions can be investigated. The wild-type TMV (wt-TMV) exhibits a negative overall charge and contains, except for tyrosine, no chemically addressable amino acids on its surface. However, by genetic engineering, a lysine mutant (TMV-Lys) (less negatively charged and with an amino group addressable by $N$-hydroxysuccinimide) and a cysteine mutant (TMV-Cys) (able to take part in thiol-Michael-type addition) have been produced. ${ }^{18}$ In addition, the E50Q mutant, which forms stable virus-like particles without incorporation of RNA but otherwise the same amino acid composition on the surface as wt-TMV, allows investigating the influence of the RNA on layer formation and mineralization (Figure 1(a)). ${ }^{19}$

A crucial point in the generation of bio/inorganic hybrids for their functional properties is the quality of the template layer. Therefore, to investigate the influence of the genetic modification on the template layer structure, wt-TMV and several TMV mutants were oriented using a convective assembly (CA) method (Figure 1(b)). ${ }^{20,21}$ Thereby, the assembly and self-organization of virus particles from dilute liquid suspensions occurs at the suspension-substrate-air contact line (Figure 1(c)). The viruses are carried by convective currents in the meniscus region due to liquid evaporation. Applying the $\mathrm{CA}$ technique, virus template layers with a controllable structure could be prepared for the subsequent mineralization step in the hybrid formation procedure. The authors' current work has incorporated TMV/zinc oxide layers in field-effect transistors (FETs). ${ }^{6,22}$ TMV/zinc oxide films used as active material in FETs showed transistor properties at room temperature without posttreatment. In addition, a newly established microwave-assisted zinc oxide deposition approach on TMV resulted in FET properties, which could be tuned by the zinc oxide layer thickness. ${ }^{22}$ Further, E50Q deposited by means of CA has shown the smoothest virus monolayers combined with the lowest surface roughness after zinc oxide mineralization. This resulted in improved FET properties compared to those of

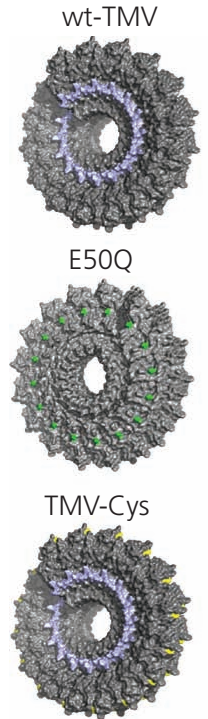

(a)
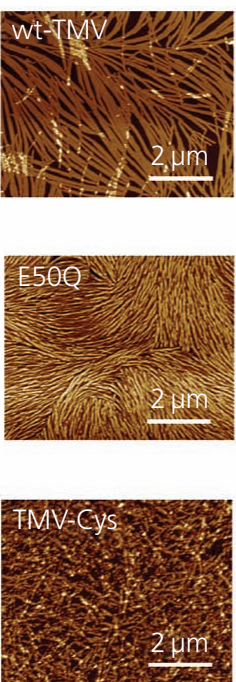

(b) (c)

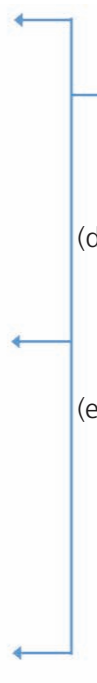

(d)
Convective assembly

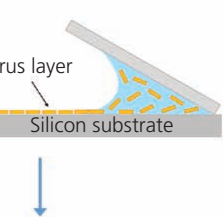

ZnO deposition

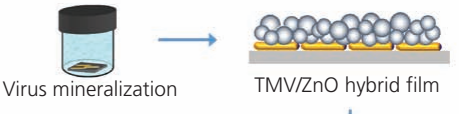

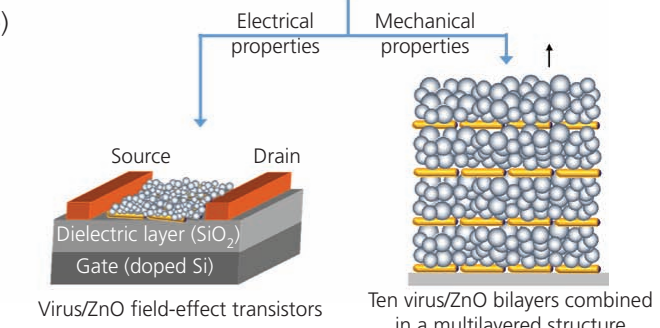

Figure 1. (a) Schematic representation of the disk structure of the not modified wt-TMV as well as E50Q and TMV-Cys mutants obtained from Protein Data Bank structure $3 J 06$ manipulated with PyMol version 1.7.4, a molecular graphics system (Schrödinger, LLC). The RNA is shown in blue, the position of the Cys mutation in yellow and the E50Q mutation in green. (b) Atomic force microscopy height images of the corresponding virus monolayers obtained through convective assembly (CA). CA conditions: $V=5 \mu \mathrm{l}, \mathrm{c}=1.9 \mathrm{mg} / \mathrm{ml}$ and withdrawal speed $=0.6 \mathrm{~mm} / \mathrm{min}$. (c) and (d) show a scheme of the synthesis pathway including template monolayer formation applying the CA technique and subsequent mineralization of the virus layer with zinc oxide at mild conditions. (e) The electrical properties of the hybrid films were measured by means of field-effect transistors and the mechanical properties of multilayered virus/zinc oxide samples were studied 
wt-TMV-based FETs. ${ }^{20}$ Recently, the intrinsic piezoelectric properties of wt-TMV, assembled in a template monolayer through CA, were used to synthesize piezoelectric zinc oxide films. They showed a twofold increase in the piezoelectric coefficient up to $7 \cdot 2 \mathrm{pm} / \mathrm{V}$ compared to films synthesized on nonpiezoelectric templates such as $\mathrm{COOH}-$ and $\mathrm{NH}_{2}-\mathrm{SAMs}^{23}$ The TMV intrinsic piezoelectricity is explained as due to the mechanical deformation of the virus when immobilized on a hydrophilic substrate. As a result, the virus height is reduced, accompanied by additional flattening of the virus structure after immersion and heating in the methanol precursor solution. Thus, the surface curvature is reduced and the parallel arrangement of the CP's intrinsic dipoles is increased. This allows well-ordered deposition of the polar zinc oxide crystals, strong enhancement of the (002) texture and subsequent increase in the zinc oxide electromechanical response. Another part of the authors' work was to understand the formation mechanism of the hybrid materials, which is essential to controlling their shape and properties. Whereas the presence of additives in the mineralization solution used for synthesis of TMV/zinc oxide hybrids obstructs the study of the deposition mechanism, the mineralization of zinc sulfide $(\mathrm{ZnS})$ on TMV was conducted without further additives and structure-directing agents. In this case, heterogeneous nucleation induced by the organic template was detected. ${ }^{24}$ So far, the influence of the zinc oxide microstructure on the properties of the TMV/zinc oxide hybrids is not understood. Accordingly, in the work presented in this paper, a mineralization pathway for the synthesis of nanocrystalline zinc oxide with tunable particle size and texture is presented. wt-TMV, the TMV mutants E50Q and TMV-Cys and amino-functionalized SAMs ( $\mathrm{NH}_{2}$-SAMs), taken as a reference, were used as organic templates. Organic/inorganic hybrid materials were prepared (Figure 1), and the electrical and mechanical properties were investigated. The authors' studies focused on the following main aspects: $(a)$ influence of zinc oxide particle size and texture on the performance of the hybridbased FETs, $(b)$ ability to form stable multilayered nacre-like zinc oxide structures making use of viruses as organic templates and (c) impact of the template/genetic modification on the electrical and mechanical properties of the hybrid materials.

\section{Results and discussion}

\subsection{Influence of water content of deposition solution on synthesis of organic/inorganic hybrid films}

Zinc oxide films for the electrical and mechanical characterization were synthesized by means of a previously established CBD process. ${ }^{5,25}$ The reaction solution comprises methanol (solvent), zinc acetate (metal oxide precursor), tetramethylammonium hydroxide (base) and polyvinylpyrrolidone (PVP; growth inhibitor). In contrast to the earlier investigations, small amounts of water $\left(\mathrm{H}_{2} \mathrm{O} ; 0-5\right.$ vol. \%) were added. This modification should facilitate the thermohydrolytic conversion of the zinc salt to zinc oxide, which would result in faster particle growth in the reaction solution and possibly also to larger crystallite sizes within the thin film after deposition. Thereby, it is assumed that nanosized zinc oxide particles form in solution first (homogeneous nucleation) and attach to the substrate in the second step.

The development of the particle size was monitored by means of dynamic light scattering (DLS). As shown in Figure 2, an increase in the water content leads to larger hydrodynamic diameters $D_{\mathrm{H}}$. Values for $D_{\mathrm{H}}$ changed from $11 \pm 3,12 \pm 2,15 \pm 2$ and $19 \pm 1$ to $23 \pm 1 \mathrm{~nm}$, corresponding to water contents of $0,1,2,3$ and 5 vol. \%, respectively. It should be taken into account that the calculation of $D_{\mathrm{H}}$ assumes spherical particles and also includes the solvent shell.

After particle size assessment of the water-containing reaction solutions, zinc oxide thin films were deposited on silicon substrates, which were previously coated with various templates that is, $\mathrm{NH}_{2}$-SAMs, wt-TMV and E50Q mutant. The SAMs were formed by immobilization of (3-aminopropyl)triethoxysilane (Aptes), while the viruses were organized in monolayers through the CA technique following a self-assembly procedure described in Section 3.5. The deposited hybrid films were characterized by means of scanning electron microscopy (SEM) and X-ray diffraction (XRD). In all cases, SEM cross-sections revealed uniform films with homogeneous thickness over the entire samples. SEM micrographs for zinc oxide films on wt-TMV as well as $\mathrm{NH}_{2}$-SAMs are shown in Figures 3 and 4(a) and 4(b). The presence of the organic templates ensured sufficient adhesion on the substrate. The layers exhibited a granular morphology with distinct mesoporosity. This is understandable as the film was composed of individual particles which were deposited from the reaction solution. Thus, the consistent attachment of the particles

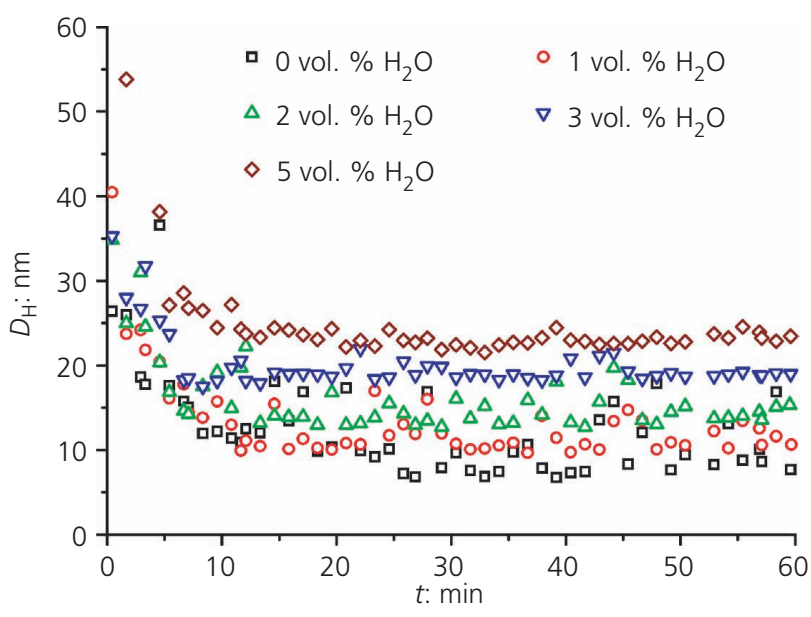

Figure 2. DLS measurements of zinc oxide precursor solutions containing different amounts of water heated at $60^{\circ} \mathrm{C}$. The determined hydrodynamic diameter $D_{H}$ is given in dependency of the measurement time $t$. Each data point corresponds to a mean value of four measurements with individually prepared reaction solutions (values for the first few minutes are included for the sake of completeness, but should be disregarded as the reaction solution has probably not reached the reaction temperature yet) 


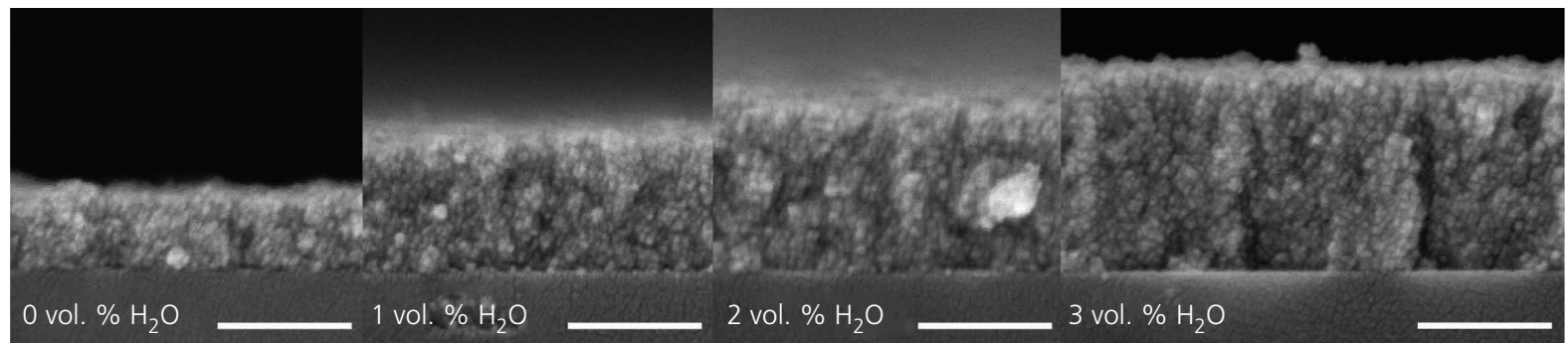

(a)

Figure 3. SEM cross-sections of thin zinc oxide films on wt-TMV monolayers produced through CA ( $c=5 \mathrm{mg} / \mathrm{ml}$ and $v_{\mathrm{w}}=1 \cdot 2 \mathrm{~mm} / \mathrm{min}$ ) after 20 mineralization cycles with (a) 0, (b) 1, (c) 2 and (d) 3 vol. \% water in the reaction solution. All scale bars represent $200 \mathrm{~nm}$

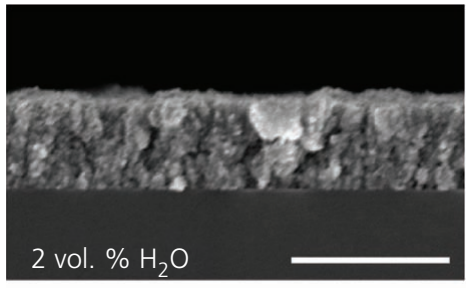

(a)

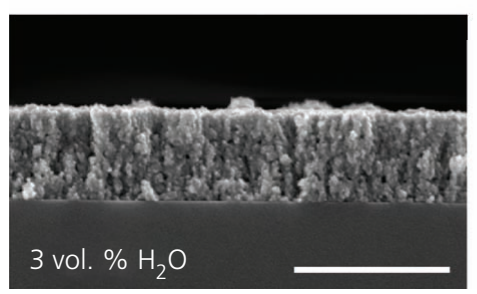

(b)

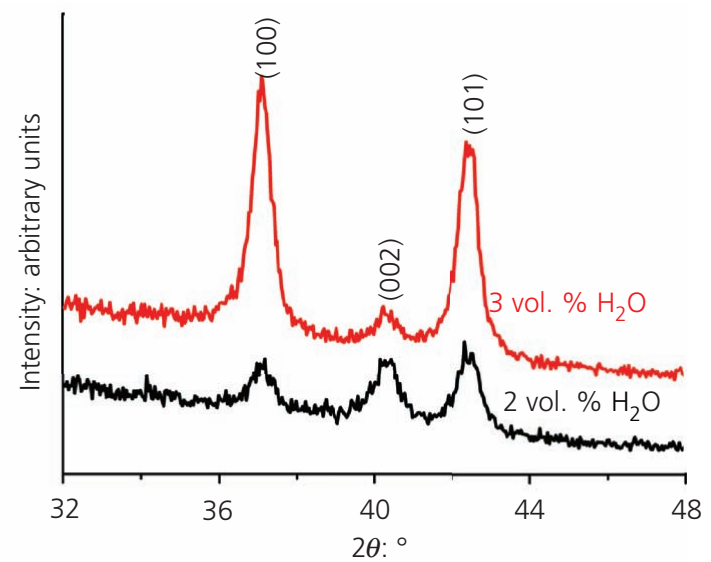

(c)

Figure 4. SEM cross-sectional images of zinc oxide films on $\mathrm{NH}_{2}$-SAMs prepared with (a) 2 vol. \% water and 20 deposition cycles and (b) 3 vol. \% water and 15 deposition cycles. The scale bars represent $500 \mathrm{~nm}$. (c) XRD patterns of the corresponding films. Taking the relative intensities of the (100) and (002) reflections into account, a moderate (002) texture is obtained with 2 vol. \% water and a (100) texture by adding 3 vol. \% water. Image (b) and the red curve in (c) were reproduced from the paper of Stitz et al. ${ }^{23}$

mineralized in solution on the previously formed oxide surface left behind some voids. The growth rate on wt-TMV-modified substrates (Table 1) increased with a rise in the water content in the reaction solution from $6 \mathrm{~nm} /$ cycle (without water) to $15 \mathrm{~nm} /$ cycle (3 vol. \% water).

XRD studies were performed on the films deposited from reaction solutions with varying water contents to confirm the formation of zinc oxide (Joint Committee on Powder Diffraction Standards card number 89-1397). Figures 4(c) and 5 reveal the formation of nanocrystalline zinc oxide. The typical three reflections ((100),

Table 1. Film thickness $t$ and the corresponding growth per cycle gc of wt-TMV/zinc oxide films after 20 mineralization cycles with varying water contents in the reaction solution ( $0-3$ vol. \% water)

$\begin{array}{lcccc}\text { Water: vol. \% } & 0 & 1 & 2 & 3 \\ t: \text { nm } & 124 \pm 4 & 198 \pm 3 & 248 \pm 3 & 306 \pm 6 \\ \text { gc, SEM: nm/cycle } & 6 & 10 & 12 & 15\end{array}$

(002) and (101)) of the hexagonal wurtzite structure of zinc oxide were observed. The broadening of the reflections indicated the formation of nanometer-sized crystallites. The crystallite sizes $\left(D_{\mathrm{XRD}}\right)$, determined by the Scherrer equation, increase from $3 \pm 1$, $7 \pm 1$ and $10 \pm 1 \mathrm{~nm}$ to $15 \pm 1 \mathrm{~nm}$ for $0,1,2$ and 3 vol. \% water, respectively, and hence confirm the accelerated hydrolysis of the precursors in the presence of small amounts of water and favor crystallite growth.

The XRD patterns in Figure 5 additionally reveal the striking fact that the texture of the formed films changes with the water content. The reflections of the film prepared without water can hardly be detected, whereas the samples prepared with 1 and 2 vol. \% water in the deposition solution show an enhanced relative intensity of the (002) reflection, compared to the ones of (100) and (101). The latter clearly indicates a preferential attachment of the crystallites in the $c$-axis direction, leading to a (002) texture. In the case of the hybrid films prepared from solutions with 3 vol. \% water, the texture is changed to (100). The 


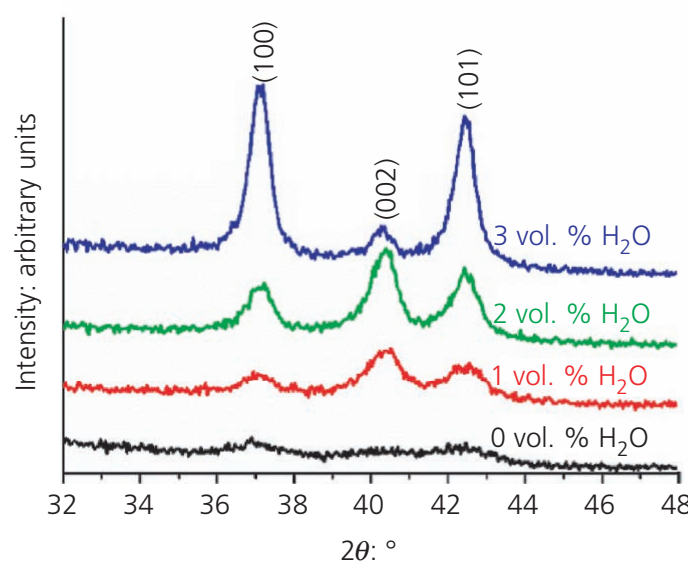

(a)

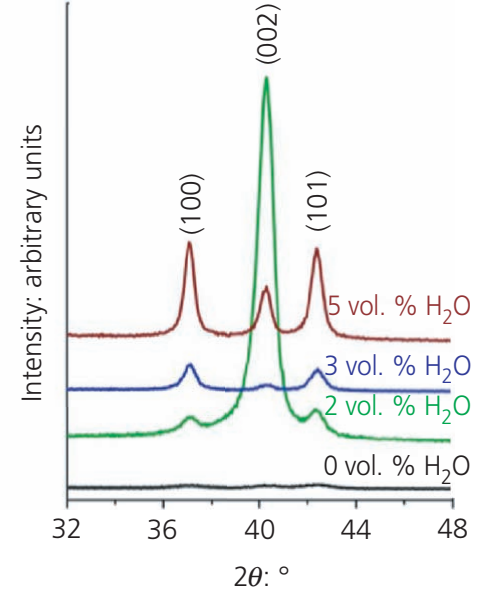

(b)

Figure 5. XRD patterns of wt-TMV/zinc oxide films obtained after (a) 20 mineralization cycles and (b) 100 mineralization cycles with different water contents. In all cases, zinc oxide is formed in the wurtzite structure. However, for 1 and 2 vol. \% water, the intensity of the (002) reflection is increased, compared to the ones of (100) and (101) diffraction lines, indicating a (002) texture of these samples. On the contrary, the relative intensity ratio indicates a (100) texture of the sample prepared with $3 \mathrm{vol}$. \% water in the reaction solution

texturing effect was more prominent with increase in the film thickness and was observed on both wt-TMV and $\mathrm{NH}_{2}$-SAM templates. At this stage, only a brief assumption about the origin of this observation could be done. Most likely, subtle differences exist in the zinc oxide particles obtained from thermohydrolysis in the reaction solution. The resulting preferential orientation might be caused by the oriented attachment of these particles on the template surface. Furthermore, this effect is more pronounced in the case of virus-based films compared to $\mathrm{NH}_{2}$-SAMs-based zinc oxide films prepared with 2 vol. \% water as seen in Figures 4(c) and 5. Hence, the influence of the template is also implied. Since the reaction was conducted in methanol solution, which restricts the dissociation of the functional groups on the template surface, it is expected that the electrostatic interactions do not govern template mineralization. Instead, the oriented attachment might be explained by the intrinsic virus piezoelectricity. ${ }^{23}$ The substrate immobilization and the methanol environment cause a strong virus deformation. As a result, the CPs in the virus structure, observed as intrinsic dipoles and arranged in a helical array, align more parallel to each other. Thus, the more pronounced dipole/ dipole interactions between the template and the zinc oxide nanocrystals could explain the enhanced texture.

\subsection{FETs of organic/inorganic hybrid films with different water contents in the deposition solution} In the following, the semiconducting properties of the aforementioned films were characterized by employment as an active layer in FETs. Since crystallite size $^{26,27}$ interface roughness, ${ }^{28}$ active layer thickness ${ }^{27,29}$ and crystallite orientation ${ }^{30}$ all have an influence on electrical performance and are all affected upon addition of water to the deposition solution, initial experiments on $\mathrm{NH}_{2}$-SAMs were carried out. The optimum water content and zinc oxide layer thickness for the best and most reproducible FET performance were determined. These findings were then transferred to virus-based transistors.

Reproducibility is a crucial point for FETs, which is pointed out also in the Institute of Electrical and Electronics Engineers standards for organic FETs. Therefore, not only a good FET performance, but also a lack of deviations in the measured values is required. To ensure reproducibility, two individual substrates, each consisting of four identical transistor units with the same channel length, were prepared applying the same deposition conditions and varying the water content. In Table 2, the FET characteristics threshold voltage $\left(V_{\mathrm{TH}}\right)$, charge mobility $(\mu)$ and on/off current ratio ( $\left.I_{\text {on/off }}\right)$ are presented as a function of the water content (2, 3 and 5 vol. \%). It was found that hybrid films, synthesized with 2 vol. \% water in the reaction solution, build FETs with the highest reproducibility of electronic characteristics.

As reported in literature, the performance of zinc oxide-based FETs is dependent on the thickness of the active semiconducting

Table 2. FET characteristics $V_{\text {Th, }} \mu$ and $I_{\text {on/off }}$ extracted from the measured output and transfer characteristics for zinc oxide films $(\approx 90 \mathrm{~nm})$ with varying water contents $(2,3$ and 5 vol. $\%$ water $)$ deposited on $\mathrm{NH}_{2}$-SAMs

\begin{tabular}{lrcc} 
Water: vol. \% & \multicolumn{1}{c}{$\boldsymbol{V}_{\text {Th }}: \mathbf{V}$} & $\boldsymbol{\mu}: \mathrm{cm}^{2} /(\mathbf{V ~ s})$ & $\boldsymbol{I}_{\text {on/off }}$ \\
\hline 2 & $10.5 \pm 0.7$ & $(1.6 \pm 1.6) \times 10^{-2}$ & $(2.0 \pm 2.5) \times 10^{6}$ \\
3 & $10.9 \pm 0.8$ & $(7.7 \pm 10) \times 10^{-3}$ & $(4.8 \pm 9.2) \times 10^{5}$ \\
5 & $5.4 \pm 4.7$ & $(4.4 \pm 5.4) \times 10^{-1}$ & $(1.5 \pm 1.5) \times 10^{6}$
\end{tabular}

The number of cycles was adjusted to eight, six and four cycles for 2, 3 and 5 vol. \% water accordingly to obtain a similar film thickness. For each condition, two individual transistor substrates were prepared and eight individual transistor units were analyzed in total. The values given are averaged over all units, and the standard deviations are given 
Table 3. FET characteristics $V_{\text {Th }}, \mu$ and $I_{\text {on/off }}$ extracted from the measured output and transfer characteristics for zinc oxide films with varying mineralization cycles $(6,8,10$ and 12$)$ deposited on $\mathrm{NH}_{2}$-SAMs from reaction solutions containing 2 vol. \% water

\begin{tabular}{lccc} 
Cycles & $\boldsymbol{V}_{\text {Th }}: \mathbf{V}$ & $\boldsymbol{\mu}: \mathrm{cm}^{2} /(\mathbf{V ~ s})$ & $\boldsymbol{I}_{\text {on/off }}$ \\
\hline 6 & $14.7 \pm 4.5$ & $(6.6 \pm 6.3) \times 10^{-3}$ & $(7.6 \pm 13) \times 10^{5}$ \\
8 & $10.5 \pm 0.7$ & $(1.6 \pm 1.6) \times 10^{-2}$ & $(2.0 \pm 2.5) \times 10^{6}$ \\
10 & $11.8 \pm 0.3$ & $(9.8 \pm 1 \cdot 1) \times 10^{-3}$ & $(6.3 \pm 0.7) \times 10^{5}$ \\
12 & $11.9 \pm 0.3$ & $(9.3 \pm 2.3) \times 10^{-3}$ & $(2.7 \pm 1.5) \times 10^{5}$
\end{tabular}

For each condition, two sets of transistors were prepared and in total eight individual transistor units were analyzed. The values given are averaged over all units, and the standard deviations are given

layer and on the method used for the zinc oxide synthesis. ${ }^{27,29}$ Therefore, in the next step, 2 vol. \% water solutions resulting in the most reproducible FETs were used to study the influence of the zinc oxide layer thickness on the FET performance of the hybrid films. As can be seen in Table 3, $\mathrm{NH}_{2}$-SAMs-templated zinc oxide films obtained after eight deposition cycles showed the highest $\mu$ and $I_{\mathrm{on} / \mathrm{off}}$ as well as the lowest $V_{\mathrm{TH}}$.

These optimized with $\mathrm{NH}_{2}$-SAMs reaction conditions were then transferred to virus-based zinc oxide FETs in order to evaluate the influence of the biotemplate on the electrical properties. As described in the aforementioned section, wt-TMV and E50Q virus monolayers were prepared through $\mathrm{CA}$ with optimized conditions for prestructured FET substrates. Figures 6(a) and 6(b) show the output characteristics for wt-TMV and E50Q-based zinc oxide FETs prepared after eight deposition cycles with 2 vol. \% water in the deposition solution and a film thickness of around $180 \mathrm{~nm}$. In both cases, a typical electronic transistor behavior without any postprocessing was demonstrated. The E50Q-based zinc oxide transistor exhibited an improved FET performance compared to wt-TMV. The output characteristics of E50Q-based zinc oxide FETs (Figure 6(b)) show a stronger current saturation compared to those of wt-TMV-based zinc oxide FETs (Figure 6(a)). Additionally, the transfer characteristics in Figure 6(c) reveal a smaller hysteresis in the case of E50Q-based zinc oxide FETs. The improved performance of the E50Q-based zinc oxide FETs is evident from the extracted characteristics $\left(V_{\mathrm{TH}}, \mu\right.$ and $\left.I_{\mathrm{on} / \mathrm{off}}\right)$, listed in Table 4. This corresponds to the results of Atanasova et $a .^{20}$ who observed an enhanced FET performance by the increased virus packing density of the E50Q mutant; thus, this enhanced performance is due to more homogeneous and almost defect-free template monolayers compared to those obtained with wt-TMV.

Then, the performances of virus-based FETs prepared without water (data taken from the paper of Atanasova et $a .^{20}$ ) and with 2 vol. \% water in the reaction solution were compared. In Figure 7, the transfer characteristics of wt-TMV (Figure 7(a)) and E50Q-based (Figure 7(b)) zinc oxide FETs are shown. The addition of water to the reaction solution leads to a reduction in $V_{\mathrm{TH}}$ from $18 \cdot 1 \pm 2 \cdot 5$ to $12 \cdot 1 \pm 1 \cdot 3 \mathrm{~V}$ in the case of wt-TMV-based zinc oxide FETs and from $14 \cdot 1 \pm 1 \cdot 7$ to $8.9 \pm 0.6 \mathrm{~V}$ in the case of E50Q-based FETs. The changes in the $\mu$ and $I_{\text {on/off }}$ values were less pronounced. In this way, charge carrier mobilities changed from $\mu 5.9 \times 10^{-4}$ to $\mu 7.7 \times 10^{-4} \mathrm{~cm}^{2} /(\mathrm{V} \mathrm{s})$ for wt-TMV and from $\mu 2.3 \times 10^{-3}$ to $\mu 2.7 \times 10^{-3} \mathrm{~cm}^{2} /(\mathrm{V}$ s) for E50Q. For the measurements carried out in this work, addition of water to the reaction solution leads to an overall better reproducibility of the properties of the resulting FETs and to a smaller hysteresis in output and transfer curves. The reason for this improvement is not easily explained, as the effect of the microstructure of a semiconducting layer on the electronic performance is complex. Larger grains within the film should reduce the number of

Table 4. FET characteristics $V_{\text {Th, }} \mu$ and $I_{\text {on/off }}$ extracted from the measured output and transfer characteristics for zinc oxide films deposited on wt-TMV and E50Q with 2 vol. \% water in the deposition solution applying eight mineralization cycles

\begin{tabular}{lrcc|} 
Virus type & \multicolumn{1}{c}{$\boldsymbol{V}_{\text {Th }}: \mathbf{V}$} & $\boldsymbol{\mu}: \mathrm{cm}^{2} /(\mathbf{V} \mathbf{~ s})$ & $\boldsymbol{I}_{\text {on/off }}$ \\
\hline Wt-TMV & $12.1 \pm 1.3$ & $(7 \cdot 7 \pm 2.6) \times 10^{-4}$ & $(1 \cdot 1 \pm 0.6) \times 10^{5}$ \\
E50Q & $8.9 \pm 0.6$ & $(2.7 \pm 0.1) \times 10^{-3}$ & $(2.2 \pm 1 \cdot 7) \times 10^{5}$
\end{tabular}

For each condition, two sets of transistors were prepared and in total eight individual transistor units were analyzed. The values given are averaged over all units, and the standard deviations are given

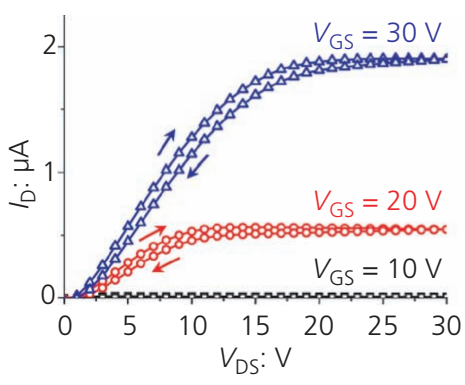

(a)

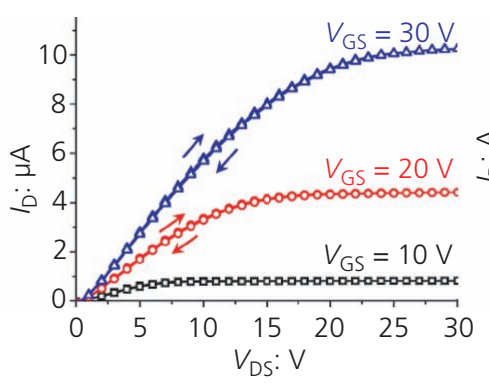

(b)

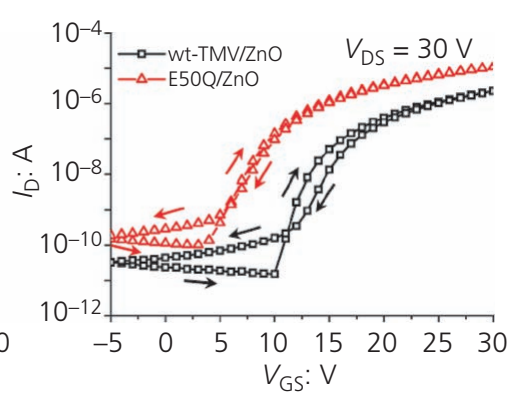

(c)

Figure 6. Output characteristics obtained from variation in the drain-source voltages $V_{D S}$ from 0 to $30 \mathrm{~V}$, for gate-source voltages $V_{\mathrm{GS}}$ varied from 0 to $30 \mathrm{~V}$ in $10 \mathrm{~V}$ steps, of (a) wt-TMV-based and (b) E50Q-based zinc oxide FETs deposited applying eight deposition cycles with 2 vol. \% water in the reaction solution. (c) Transfer characteristics for constant drain-source voltage $V_{D S}$ at $30 \mathrm{~V}$ 


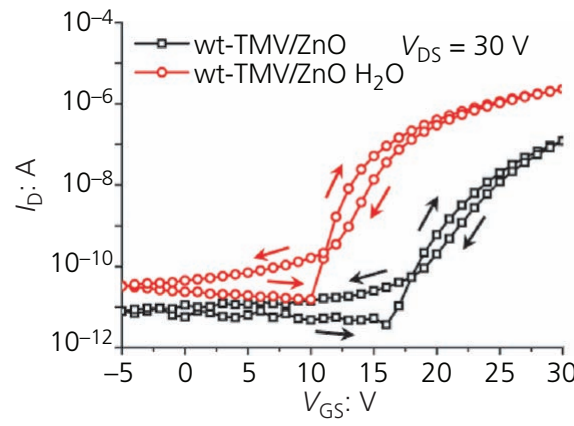

(a)

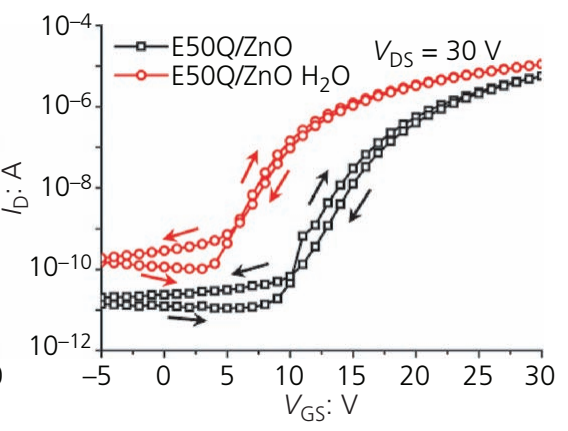

(b)

Figure 7. Transfer characteristics for constant drain-source voltage $V_{D S}$ at $30 \mathrm{~V}$. Comparison of (a) wt-TMV-based zinc oxide FETs prepared without water (wt-TMV/zinc oxide) and with 2 vol. \% water (wt-TMV/zinc oxide water). (b) E50Q-based zinc oxide FETs prepared without water (E50Q/zinc oxide) and with 2 vol. \% water (E50Q/zinc oxide water)

resulting boundaries and thus should be advantageous for the semiconducting layer in FETs. ${ }^{31,32}$ However, on the contrary, films consisting of very small crystallites might exhibit a higher packing density due to smaller voids and thus a better electronic performance. $^{33,34}$

\subsection{Mechanical properties of virus/zinc oxide multilayers}

Nacre is a natural material with extraordinary mechanical properties. It consists of $95 \mathrm{wt}$. \% calcite (calcium carbonate $\left.\left(\mathrm{CaCO}_{3}\right)\right)$ in the form of aragonite platelets embedded in $5 \mathrm{wt}$. \% protein matrix. ${ }^{35,36}$ Each of the features in the nanostructured 'brick-and-mortar' architecture contributes to the toughening of the material. It has not only a doubled strength due to its layered structure, ${ }^{37}$ but also an octuplicated fracture toughness compared to monolithic calcium carbonate ${ }^{38}$ resulting from enhanced crack deflection being one of the main toughening mechanisms. ${ }^{39}$ Nacre-like structured composites have been successfully synthesized with inorganic materials, such as titanium dioxide $\left(\mathrm{TiO}_{2}\right),{ }^{40,41}$ aluminum oxide $\left(\mathrm{Al}_{2} \mathrm{O}_{3}\right),{ }^{42}$ zinc oxide, ${ }^{43-45}$ zirconium dioxide $\left(\mathrm{ZrO}_{2}\right)^{46}$ and magnetite. ${ }^{47}$ The organic counterparts in these cases were mostly polymers. Recently, genetically engineered phages, expressing an inorganic-binding peptide for molecular recognition of zinc oxide, were used as templates to produce multilayered structures (MLSs). Mechanical tests have shown the genetically controlled influence of the bioorganic phase on the macroscopic mechanical properties of the MLS. Here, making use of TMV as a biotemplate with unique structural dimensions (Figure 1) and stability, nacre-like TMV-based zinc oxide MLSs were synthesized and their mechanical properties were studied. It is well known that nacre has an optimum thickness ratio of $1: 10$ of the organic to the inorganic phase, which is responsible for its fracture toughness, combined with stiffness and strength. In order to maintain this organic/inorganic ratio in the authors' virus-based MLS, the synthesis conditions had to be previously adjusted. In the preliminary mechanical tests, samples prepared with 3 vol. \% water in the deposition solution have shown the best results. A schematic representation of the
MLS synthesis procedure is shown in Figure 1(e). Shortly, virus monolayers were assembled through $\mathrm{CA}$ and mineralized with zinc oxide from 3 vol. $\%$ water solutions and applying six deposition cycles. This procedure was repeated until ten such organic/inorganic bilayers were obtained. As references, the corresponding monolithic samples were prepared. They lack organic interlayers and consist of only one TMV starting layer and one zinc oxide film which is formed by 60 consecutive deposition cycles. wt-TMV, E50Q and TMV-Cys mutants were chosen as virus templates. E50Q has the same surface chemistry as wt-TMV, but, due to the lack of RNA in the inner channel and its higher flexibility, it builds much more homogeneous and dense monolayers. Therefore, comparison of both systems, wt-TMV/ zinc oxide and E50Q/zinc oxide MLSs, could reveal whether the difference in the template structuring alone can influence the mechanical properties. As already mentioned, the TMV-Cys mutant has thiol groups on its outer surface, which can result in interparticle disulfide bridges. This is the reason for entangled and out-of-plane virus layers while applying the same CA conditions as for the other virus types. ${ }^{20}$ Thus, comparing the TMV-Cys/zinc oxide MLS with wt-TMV/zinc oxide MLS, two possible factors by means of structuring effect but also genetic modification coming from the cysteine residues on the virus surface might play a role and influence their mechanical properties.

In Figure 8, the corresponding SEM cross-sectional images of the MLS and their monolithic references are shown. The reference samples of each mutant show comparable monolithic structures, while the MLSs of wt-TMV, E50Q and TMV-Cys reveal the successful mimicking of nacre's brick-and-mortar structure.

The zinc oxide layer thickness was homogeneous throughout the inorganic phase. Local delamination between the layers caused by sample preparation can be seen. However, due to the strong attachment of the first virus layer to the substrate and the subsequent zinc oxide layer, both the MLS and the monolithic references are robust composite films with good adherent properties. 
Bioinspired, Biomimetic and Nanobiomaterials Volume 8 Issue BBN1
Engineered nanostructured virus/ZnO

hybrid materials with dedicated

functional properties

Atanasova, Hoffmann, Stitz et al.

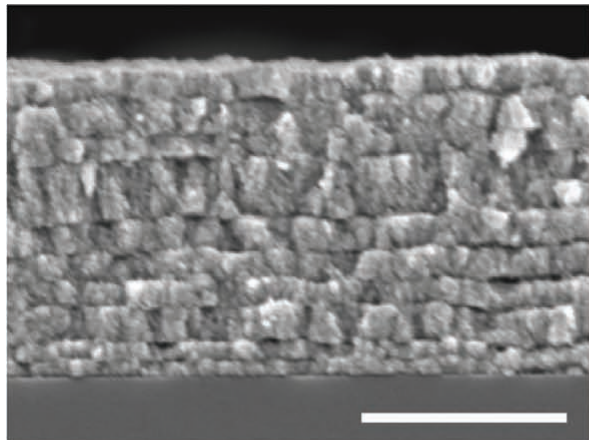

(a)

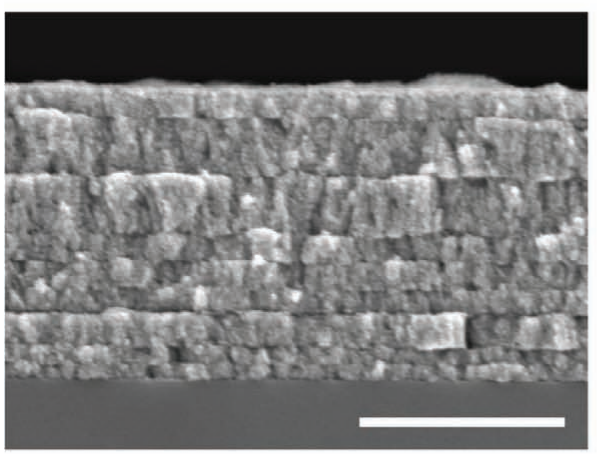

(c)

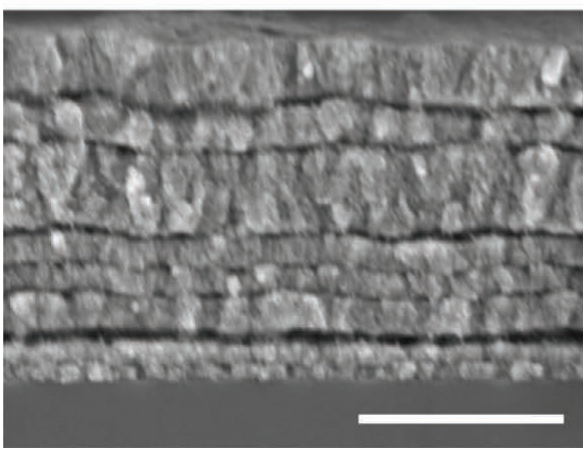

(e)

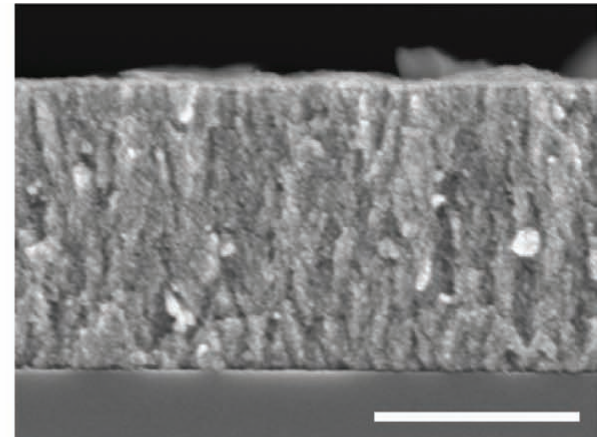

(b)

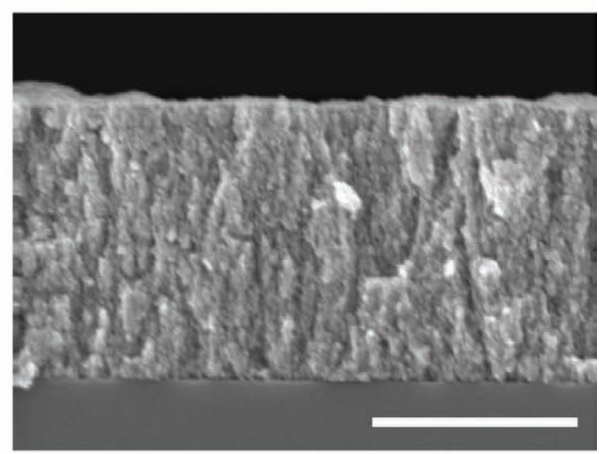

(d)

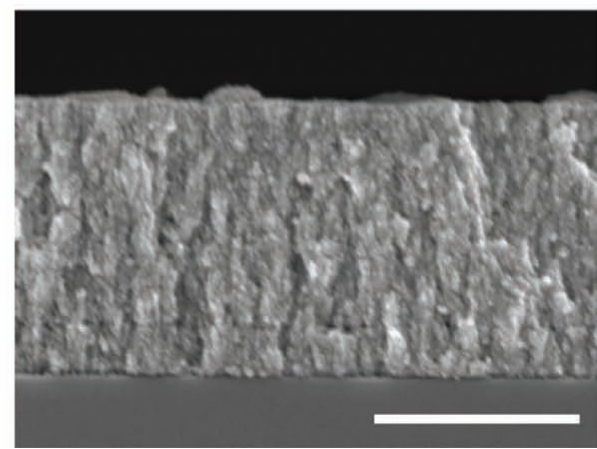

(f)

Figure 8. SEM cross-sections of the multilayered samples (MLS, left) and the corresponding monolithic reference sample (right) obtained with 3 vol. \% of water in the reaction solution: (a, b) wt-TMV/zinc oxide; (c, d) E50Q/zinc oxide; (e, f) TMV-Cys/zinc oxide. The scale bars represent $800 \mathrm{~nm}$

The hardness and Young's modulus of the MLSs and the corresponding reference samples were determined by nanoindentation, and the calculated values are summarized in Figure 9.

For the wt-TMV-based samples, the measurements revealed for both hardness and Young's modulus lower values for the MLS compared to the monolithic reference. The hardness decreased by $\approx 40 \%$ for the MLS and the Young's modulus by $\approx 30 \%$. Similar results were found for E50Q-based samples. Here, evidently the difference in the structure of the template layers (wt-TMV and E50Q) did not have an impact on the mechanical performance. The interaction of the virus interlayers, possessing the same surface groups, with the zinc oxide is poor, resulting in a sliding of the zinc oxide layers on the TMV layer upon deformation. Thus, the hardening mechanism of energy dissipation in the organic layer is not being applied. However, the performance of the TMV-Cys-based MLS differed from the wt-TMV and E50Q MLSs. The hardness increased by a factor of $\approx 27 \%$ and Young's modulus by $10 \%$ upon multilayering. According to the Reuss model of the rule of mixtures, the combination of a stiff inorganic and a soft organic material leads to a reduced Young's modulus of the resulting composite, and it is valuable for wt-TMVand E50Q-based MLSs. However, the enhanced Young's modulus of the TMV-Cys MLS contradicts this theory, but it is in good accordance with the results found for MLS thin films composed of polyelectrolytes alternated with zinc oxide ${ }^{43}$ and titanium dioxide. ${ }^{41}$ This behavior can be explained by the enhanced interaction between organic and inorganic phases at the interface in the case of the 
Bioinspired, Biomimetic and Nanobiomaterials Volume 8 Issue BBN1
Engineered nanostructured virus/Zno hybrid materials with dedicated

functional properties

Atanasova, Hoffmann, Stitz et al.

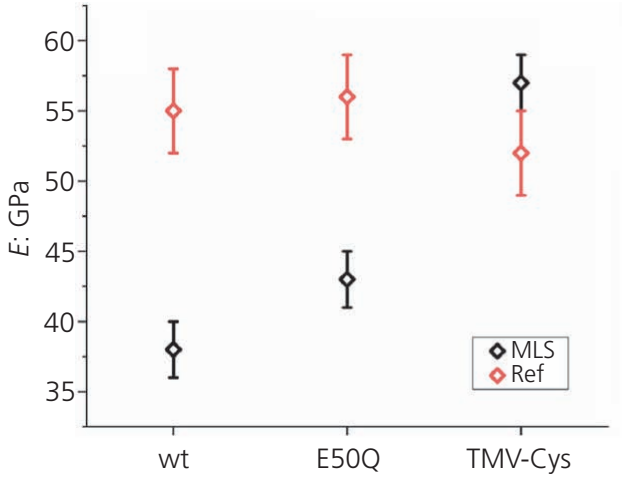

(a)

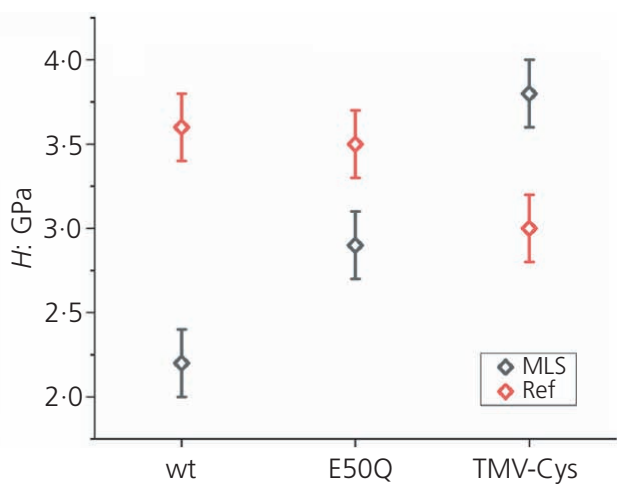

(b)

Figure 9. (a) Young's modulus $E$ and (b) hardness $H$ of the MLSs and monolithic references (Ref) as a function of the template

TMV-Cys MLS as a result of stronger affinity of cysteine toward zinc oxide. ${ }^{48,49}$ The hardness increase of the TMV-Cys MLS cannot be attributed to periodical variation of soft and stiff materials as suggested by Koehler ${ }^{50}$ and Fratzl et al. ${ }^{51}$ since both wt-TMV and E50Q also form well-distinguished MLSs despite showing a hardness decrease. Therefore, the influence of the genetic modification of the virus on the hardness enhancement of the TMVCys MLS is implied. The entangled and out-of-plane virus particles, caused by the genetic modification, penetrate into the subsequently deposited inorganic phase, and, together with the enhanced affinity of this mutant toward zinc oxide, this facilitates TMV-Cys/zinc oxide and TMV-Cys/TMV-Cys interactions. The latter increases the force necessary to drive the field of deformation through the MLS, causing an increased hardness. In Figures 10(a) and 10(c), the crack deflection in the MLSs compared to the brittle fracture of the corresponding references (Figures 10(b) and 10(d)) can clearly be seen. The organic interlayers present in the MLS cause the crack to deflect, blunt and branch. All these effects lead to an enhanced energy dissipation compared to the straight crack through the monolayer here used as a reference, which should lead to enhanced

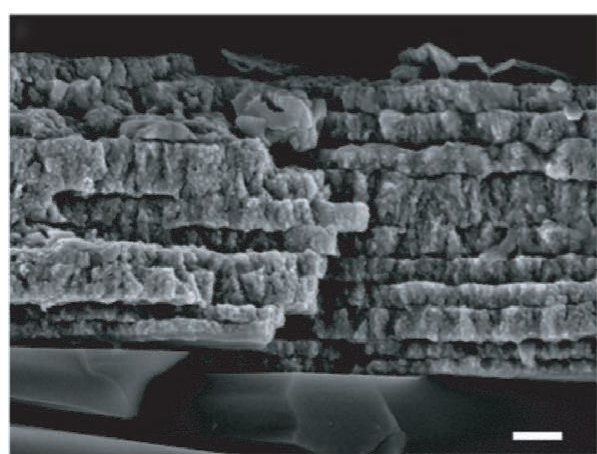

(a)

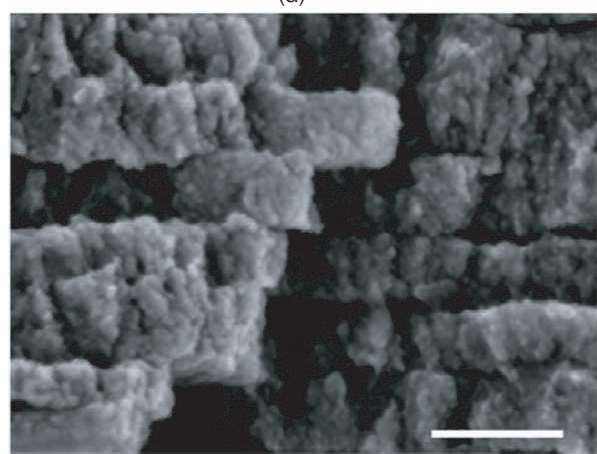

(c)

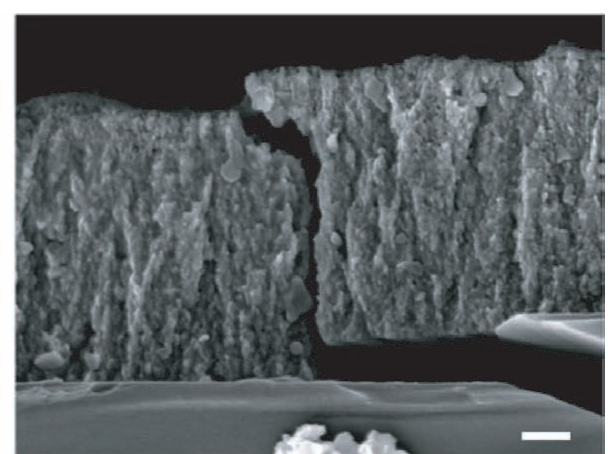

(b)

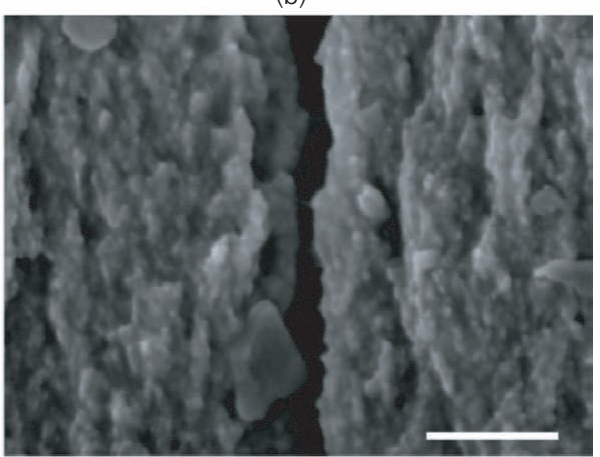

(d)

Figure 10. Cross-sectional SEM images (lower and higher magnifications) of ( $a, c)$ MLSs and (b, d) monolithic references revealing crack propagation through the films. All scale bars are $200 \mathrm{~nm}$ 
fracture toughness. Additionally, a pullout of cracked zinc oxide layers is observable, which is comparable to the platelet pullout in nacre. The inherent surface roughness of the nanocrystalline zinc oxide layers resembles the nanoasperities present on the surface of aragonite platelets in nacre, inducing friction upon their pullout. The morphology of the individual zinc oxide layers consisting of nanocrystallites is similar to the nanostructure of the individual aragonite platelets in nacre. Thus, it can be expected that rotation and deformation of such nanocrystallites contributes to energy dissipation as described for nacre. ${ }^{52}$

In order to assess the fracture toughness of MLSs and references, a thin-film approach developed by Xia et al. ${ }^{53}$ was used. Microindents were done in partially covered samples, and the crack length in the substrate $(b)$ was compared with the crack length in the coating $(a)$. The main finding is that cracks $a$ in the reference samples are always longer than cracks $b$, whereas cracks $a$ in the MLS are always shorter than the cracks $b$. Thus, more energy is dissipated in the MLSs than in the references, which proves the enhancement of the fracture toughness $K_{\mathrm{c}}$ in the case of the MLSs compared to the references for both wt-TMV and TMV-Cys. The quantitative evaluation of fracture toughness neglecting the contribution of residual stresses - was performed only for multilayered samples, since the references did not fulfill the requirement of $b>a ;^{53}$ thus, the formula is not valid for the references. However, it is possible to state that the fracture toughness of the references does not exceed the value for the silicon substrate. Thus, the fracture toughness of the references can be maximally $K_{\mathrm{c}, \text { ref }}=0.83 \mathrm{MPa} \mathrm{m}^{1 / 2}$. The obtained fracture toughness values of the MLSs are $K_{\mathrm{c}, \mathrm{wt}-\mathrm{TMV}}=1.8 \pm 0 \cdot 4 \mathrm{MPa} \mathrm{m}^{1 / 2}$ and $K_{\mathrm{c}, \text { Cys }}=1 \cdot 3 \pm 0 \cdot 4 \mathrm{MPa} \mathrm{m}^{1 / 2}$. Even though no absolute values could be calculated for the reference monolayer films, the enhancement of the fracture toughness, by mimicking nacre's hierarchical structure, is at least $+54 \%$ for wt-TMV and $+36 \%$ for TMV-Cys. The absolute calculated value of $K_{\mathrm{c}, \text { Cys }}$ is smaller than that in the case of wt-TMV. This corresponds to the enhanced hardness and same Young's modulus of TMV-Cys/zinc oxide composites compared to those of the reference. The enhanced interaction of the cysteine groups with zinc oxide leads to a stronger connection between the organic and inorganic phases, allowing less energy dissipation, highlighting the importance of the interaction between the organic and inorganic phases.

\section{Materials and methods}

\subsection{Virus genetic modification and purification}

wt-TMV and TMV-Cys ${ }^{18}$ were propagated in Nicotiana tabacum Samsun nn in a greenhouse and purified according to the protocol of Gooding and Hebert. The E50Q mutant ${ }^{19}$ was purified from Nicotiana benthamiana using the protocol described by Atanasova et al. ${ }^{20}$

\subsection{DLS measurements}

DLS experiments were performed on a Malvern Zetasizer 3000HS equipped with a helium $(\mathrm{He})$-neon $(\mathrm{Ne})$ laser with a wavelength of
$633 \mathrm{~nm}$ and $90^{\circ}$ detection. Measurements were carried out at $60^{\circ} \mathrm{C}$. The viscosity of the reaction solution at $60^{\circ} \mathrm{C}$ was determined with a RheoStress 1 instrument (Thermo Haake, rotor DG43 titanium (Ti) and beaker DG42) with a constant shear rate $\gamma$ of $300 \mathrm{~s}^{-1}$. The measurement duration was $60 \mathrm{~s}$, and the viscosity was evaluated ten times, resulting in a value of $0.76 \mathrm{mPa} \mathrm{s}$. The refractive index of the reaction solution was measured with a refractometer as $n_{\mathrm{RS}}=$ 1.328. The real and imaginary parts of the refractive index of the formed zinc oxide were obtained from the manufacturer's manual $\left(n_{\mathrm{R}}=2 \cdot 0\right.$ and $\left.n_{\mathrm{I}}=0 \cdot 1\right)$. Measurements of reaction solutions with different water contents were repeated at least three times and measured without subruns, collecting data points every $15 \mathrm{~s}$. The data points were averaged over four individual measurements with fresh solution, and the standard deviation was determined. For the generation of the plots of the hydrodynamic diameter in dependency of the time, three consecutive data points were averaged to smooth the plot.

\subsection{Substrate preparation}

Silicon substrates (100, p-doped polished wafers, Silchem, Germany) were cleaned by sonication successively in water and ethanol/acetone $(1: 1 \mathrm{v} / \mathrm{v})$ for $10 \mathrm{~min}$. Then, a plasma cleaning step $(10 \mathrm{~min} 30 \mathrm{~W})$ was performed to hydrophilize the substrates followed by $10 \mathrm{~min}$ sonication in water. The substrates were washed ten times with the corresponding solvent after each sonication step and dried with inert gas.

FET substrates were cleaned prior to use as follows: $5 \mathrm{~min}$ sonication sequentially in acetone, isopropanol and ultrapure water; 1 min plasma cleaning $(70 \mathrm{~W})$; and $10 \mathrm{~min}$ sonication in ultrapure water. After each sonication step, the substrates were washed with the corresponding solvent and dried with inert gas.

\subsection{SAM preparation}

Aptes (99 vol. \%, Acros Organics) was deposited from a 2 vol. \% solution in ethanol/water $(95: 5 \mathrm{v} / \mathrm{v})$ in order to functionalize the cleaned silicon substrates with an amino-terminated SAMs. The solution was left $5 \mathrm{~min}$ to hydrolyze. Then, cleaned silicon substrates were placed in Aptes solution for $1 \mathrm{~h}$ at room temperature. Subsequently, the functionalized wafers were thoroughly washed several times with ethanol and dried under inert gas. ${ }^{54}$

\subsection{Convective assembly}

Homogeneous virus monolayers of wt-TMV, E50Q and TMV-Cys mutants were obtained by CA as described in detail by Atanasova et $a .^{20}$ and Atanasova. ${ }^{21}$ The procedure will be outlined shortly here. Cleaned silicon substrates $\left(15 \times 15 \mathrm{~mm}^{2}\right)$ were mounted on a substrate holder in a glass chamber (constant temperature and humidity) and connected to a horizontally positioned linear motor (dip coater, KSV Instruments, Espoo, Finland) outside the chamber. The glass deposition plate $\left(14 \times 40 \mathrm{~mm}^{2}\right)$ was fixed under a certain angle to the silicon substrate, and a droplet of the virus solution $(5 \mu \mathrm{l})$ was placed between them, resulting in a meniscus. All virus assemblies were performed at $23 \pm 2{ }^{\circ} \mathrm{C}$ and a 
humidity of $35 \pm 2 \%$ relative humidity. The concentration of the viruses dispersed in ultrapure water was $1.9 \mathrm{mg} / \mathrm{ml}$ for all virion types, and a withdrawal speed of $0.6 \mathrm{~mm} / \mathrm{min}$ was used.

\subsection{Zinc oxide deposition solution and mineralization}

Stock solutions of zinc acetate dihydrate $\left(\mathrm{Zn}\left(\mathrm{OOCCH}_{3}\right)_{2} \cdot 2 \mathrm{H}_{2} \mathrm{O}\right)$ $\left(\mathrm{ZnAc}_{2}\right)$ (Sigma-Aldrich), PVP (Sigma-Aldrich, Mw $=10$ 000, lot number BCBJ4889V) and tetraethylammonium hydroxide (TEAOH, Aldrich Chemistry) dissolved in methanol were prepared with concentrations as follows: $34 \mathrm{mM}\left(\mathrm{ZnAc}_{2}\right)$, $21.7 \mathrm{mM}$ (PVP) and $75 \mathrm{mM}$ (TEAOH). Prior to use, the deposition solution was always freshly prepared out of the three stock solutions. One volume unit of the $\mathrm{ZnAc}_{2}$ stock solution and one volume unit of the PVP stock solution were mixed. Then, the same volume unit of the TEAOH stock solution was added dropwise by using a peristaltic pump at a flow rate of $1.04 \mathrm{ml} / \mathrm{min}$ under continuous stirring. Thus, final concentrations of $\left[\mathrm{Zn}^{2+}\right]=$ $11.3 \mathrm{mM},[\mathrm{PVP}]=7 \cdot 2 \mathrm{mM}$ and $[\mathrm{TEAOH}]=25 \mathrm{mM}$ were obtained. One milliliter deposition solution was used for the mineralization. Therefore, in order to obtain the required water content, prior to use, small amounts of water were added to the deposition solution (e.g. for 2 vol. \%, $980 \mu 1$ deposition solution and $20 \mu \mathrm{l}$ ultrapure water). Then, silicon substrates with immobilized viruses were placed in this solution and heated at $60^{\circ} \mathrm{C}$ for $90 \mathrm{~min}$ to complete one mineralization cycle. Subsequently, substrates were washed thoroughly with methanol and dried. The next mineralization cycle was performed accordingly with freshly mixed water deposition solution.

\subsection{Synthesis of multilayered samples and monolithic references}

The multilayered samples (MLSs) were prepared by combining CA of TMV in template layers with zinc oxide deposition. Silicon substrates were functionalized with wt-TMV, E50Q and TMV-Cys monolayers by $\mathrm{CA}$ as described earlier. The number of zinc oxide mineralization cycles was chosen to achieve a thickness ratio of $1: 10$ of the organic to the inorganic phase. Silicon substrates functionalized with viruses were mineralized six times, and then the silicon substrate was rotated $90^{\circ}$ and CA for immobilization of the next virus layer was performed. The entire process was repeated ten times. Thus, ten organic/inorganic bilayers were obtained.

For the monolithic reference samples, only the first virus layer was deposited followed by the same number of zinc oxide mineralization cycles (60 cycles). Finally, for all samples, the starting layer is always an organic layer and the final layer is an inorganic layer.

\subsection{Characterization}

Atomic force microscopy on a Digital Instruments MultiMode 8 from Bruker with a NanoScope 5 controller operated in the tapping mode was used to image the virus layers. Silicon cantilevers and PPP-NCHR-W (Nanosensors) $\mathrm{n}+$-doped tips with resistivity of $0.01-0.02 \Omega \mathrm{cm}$ were used.
SEM was performed on a field emission Zeiss DSM982 Gemini microscope with a thermal Schottky field emitter equipped with an in-lens detector. The microscope was operated at an acceleration voltage of $3 \mathrm{kV}$ and a working distance of $3 \mathrm{~mm}$.

Samples were sputtered with $0.5 \mathrm{~nm}$ platinum-palladium $(80: 20)$ alloy.

XRD measurements were conducted on a Panalytical X'Pert MRD instrument equipped with a cobalt (Co) tube and polycapillary optics, an Eulerian cradle, a diffracted beam monochromator and a scintillation counter. Symmetrical $\theta-2 \theta$ scans were performed covering the reflections (100), (002) and (101). The specimen was tilted to an angle of $\chi=10^{\circ}$ using the cradle in order to avoid detection of diffracted intensity from the silicon substrate crystal.

\subsection{Electrical characterizations}

FET substrates were purchased from the Fraunhofer Institute for Photonic Microsystems, Dresden, Germany. These substrates consist of a highly n-doped $\left(n \sim 3 \times 10^{17} \mathrm{~cm}^{-3}\right)$ silicon substrate with a thermally grown $90 \mathrm{~nm}$ layer of silicon dioxide $\left(\mathrm{SiO}_{2}\right)$. The source-drain electrodes are prefabricated $30 \mathrm{~nm}$ gold electrodes, containing a $10 \mathrm{~nm}$ intermediate adhesive layer of indium tin oxide. The electrodes have an interdigital pattern, possessing a channel width of $10 \mathrm{~mm}$ and a channel length of $10 \mu \mathrm{m}$. The electrical characterization was performed in a glove box with an HP 4155A semiconductor parameter analyzer (Agilent), under an argon atmosphere. Output characteristics of all FEts were obtained by varying the drain-source voltage from 0 to $30 \mathrm{~V}$ for gate-source voltages from 0 to $30 \mathrm{~V}$ in $10 \mathrm{~V}$ steps. Transfer characteristics were measured for a constant drain-source voltage of $30 \mathrm{~V}$ and varying gate-source voltages from -5 to $30 \mathrm{~V}$. Mobility values have been extracted performing a linear fit to the square root of the drain current from the transfer characteristic. Further details of the extraction parameters have been reported previously. $^{6}$

\subsection{Nanoindentation}

Measurements were conducted in the continuous stiffness measurement mode. Thus, the indenter tip was constantly driven into the sample while applying an additional harmonic load. A sharp cube corner indenter (tip radius $\approx 40 \mathrm{~nm}$ ) with reduced sensitivity against the roughness of the film surface was used, creating plastic deformation within small indentation depths. Therefore, the field of deformation (combination of elastic and plastic deformation) remains within the film, leading to less substrate influence. Nanoindentation data were evaluated according to the method described by Oliver and Pharr ${ }^{55}$ in the contact depth range between 10 and $20 \%$ of the total film thickness to avoid influences of the surface roughness and the substrate. ${ }^{56}$ Twenty-five indents were performed with a distance of $10 \mu \mathrm{m}$ at three different places to obtain good statistics and to characterize the samples over a large area on every sample. All given values are the mean values of these indents. 


\subsection{Synthesis of samples for fracture toughness assessment}

Multilayered samples used for fracture toughness analysis consisted of five organic/inorganic bilayers. The monolithic references were synthesized by zinc oxide mineralization of the corresponding starting virus template layer with 30 deposition cycles. To obtain the partially covered samples necessary for fracture toughness evaluation according to Xia et al. ${ }^{53}$ a photoresist layer was applied in order to protect part of the zinc oxide film, while the rest was etched to reach a bare silicon substrate. Therefore, samples were spin coated for $30 \mathrm{~s}$ at 4500 revolutions/min with photoresist S1805 (Shipley Company) followed by baking at $90^{\circ} \mathrm{C}$ on a hot plate for $2 \mathrm{~min}$ and exposing for $40 \mathrm{~s}$ with Maskligner MA6. Chrome masking was performed in such a way that the larger part of the samples was covered. Samples were developed with developer AZ 726 MIF (AZ Electronic Materials $\mathrm{GmbH}$ ) for $30 \mathrm{~s}$, washed with a large amount of water and dried with nitrogen gas $\left(\mathrm{N}_{2}\right)$. Masked samples were dipped into $0.5 \mathrm{M}$ hydrochloric acid $(\mathrm{HCl})$ until the unprotected part of the sample was wetted to dissolve the zinc oxide films (reference and multilayer) from the unprotected part of the substrate. Following that, samples were washed with water and dried with nitrogen gas. In the next step, the samples were then completely immersed in acetone to remove the photoresist and dried with nitrogen gas. Thus, a sharp edge between the bare silicon substrate and the film was obtained.

\subsection{Determination of fracture toughness}

The fracture toughness of the samples was determined by microindentation with a Leica VMHT MOT hardness tester according to the method published by Xia et al. ${ }^{53}$ Radial cracks arise upon indentation in the uncoated substrate region close to the coating. Subsequently, the crack length is detected with varying loads. Fracture toughness is then estimated by comparing the lengths of the cracks that propagate into $(a)$ and away $(b)$ from the film. The applied loads for the indents were 0.3 and $0 \cdot 5 \mathrm{~kg}$. The crack length was measured using a light microscope. Specifically, the fracture toughness $K_{\mathrm{c}}$ of the film can be estimated from the measured crack length on the substrate $(b)$ and within the coating $(a)$, according to the equation

$$
\begin{aligned}
K_{\mathrm{c}}= & \left\{K_{\mathrm{s}}^{2}\left[1+\lambda \frac{(\phi b-a)}{t} \sqrt{\frac{E_{\mathrm{c}}\left(1-v_{\mathrm{s}}^{2}\right)}{E_{\mathrm{s}}\left(1-v_{\mathrm{c}}^{2}\right)}}\right]^{2}\right. \\
& \left. \pm\left[2 \psi_{\mathrm{c}} \sigma_{\mathrm{r}} \sqrt{t} \sqrt{\frac{E_{\mathrm{c}}\left(1-v_{\mathrm{s}}^{2}\right)}{E_{\mathrm{s}}\left(1-v_{\mathrm{c}}^{2}\right)}}\right]^{2}\right\}^{1 / 2}
\end{aligned}
$$

where the dimensionless factors $\lambda$ and $\psi_{\mathrm{c}}$ are 0.45 and 0.95 , respectively, and $t$ is the film thickness, which was determined through cross-sectional SEM images. The formula was derived for the condition that the crack in the coating $(a)$ is always shorter than the crack in silicon $(b)$. Thus, the equation is not valid for $a>b .^{53}$ If $a>b$, the fracture toughness cannot be calculated exactly, but is limited by the fracture toughness of the silicon substrate $K_{\mathrm{s}}$. For the fracture toughness $K_{\mathrm{s}}$, Young's modulus $E_{\mathrm{s}}$ and Poisson's ratio $v_{\mathrm{s}}$ of the silicon substrate, the respective literature values of $0.83 \mathrm{MPa} \mathrm{m} \mathrm{m}^{1 / 2}, 163 \mathrm{GPa}$ and 0.22 were used. ${ }^{53}$ For the Young's modulus of the films $E_{\mathrm{c}}$, values obtained by nanoindentation were used, while a Poisson's ratio $v_{\mathrm{c}}$ of $0 \cdot 25$ was used. The dimensionless factor $\phi$ was calculated from the slope of the dependence of the substrate crack length against the crack length in the film. The residual stress in the film $\sigma_{\mathrm{r}}$ was neglected in this case. The standard deviation of the fracture toughness $\Delta K_{\mathrm{c}}$ was estimated by applying the law of error propagation, which leads to the equation

$$
\begin{aligned}
& \Delta K_{\mathrm{c}} \\
& =\sqrt{\left(\frac{\partial K_{\mathrm{c}}}{\partial E_{\mathrm{c}}} \Delta E_{\mathrm{c}}\right)^{2}+\left(\frac{\partial K_{\mathrm{c}}}{\partial a} \Delta a\right)^{2}+\left(\frac{\partial K_{\mathrm{c}}}{\partial b} \Delta b\right)^{2}+\left(\frac{\partial K_{\mathrm{c}}}{\partial t} \Delta t\right)^{2}}
\end{aligned}
$$$$
2 .
$$

Equation 1 is partially derived for each measured variable. The $\Delta$ values are the standard deviations obtained when determining each of the error-prone variables $\left(E_{\mathrm{c}}, t, a\right.$ and $\left.b\right)$.

\section{Conclusion}

The influence of the microstructure of organic/inorganic hybrid materials (thin films of nanocrystalline zinc oxide on substrates modified with various types of TMVs or SAMs) on the corresponding electronic and mechanical properties was systematically studied. The texture and grain size of the zinc oxide moiety in these hybrids could be adjusted by altering the water content in the deposition solution of the chemical bath process. Thus, films with 3 and 5 vol. \% water showed a (100) texture, whereas 1 and 2 vol. \% water lead to a (002) texture. The texture not only was more pronounced in the case of an increase in film thickness but was also affected by different templates. The texture obtained on wt-TMV was strongly increased compared to the ones on $\mathrm{NH}_{2}$-SAMs used as a reference hybrid structure. The reason for this finding can be explained based on the intrinsic virus piezoelectricity. ${ }^{23}$ The more parallel dipole (CP) alignment contributes to improved dipole/dipole interactions between the virus and the zinc oxide nanocrystals and leads to enhancement in the texture of the TMV surface. The influence of the water content in the deposition solution and the texture effect, respectively, was studied also on the FET performance of the hybrid films. Hereby, zinc oxide layers deposited on $\mathrm{NH}_{2}$-SAMs and wt-TMV as well as on the E50Q mutant were compared. The best and most reproducible FET results were obtained with films prepared by the addition of 2 vol. \% water to the reaction solution. In comparison to earlier reports, ${ }^{20}$ the addition of water is in all cases certainly beneficial and is able to increase charge carrier mobilities by one or two orders of magnitude and furthermore to reduce the threshold voltage. Differences in FET performance of zinc oxide layers on wt-TMV and E50Q are attributed to the influence of the quality (more homogeneous and dense E50Q monolayer) induced by the genetic modification rather than electronic interactions between 
Bioinspired, Biomimetic and Nanobiomaterials Volume 8 Issue BBN1
Engineered nanostructured virus/ZnO

hybrid materials with dedicated

functional properties

Atanasova, Hoffmann, Stitz et al. virions and zinc oxide. Mechanical properties of the hybrid films were quantified by means of nanoindentation. Based on preliminary experiments, a 3 vol. \% water-containing deposition solution was used to prepare nacre-like virus-based zinc oxide multilayers. While the mechanical testing on the wt-TMV and E50Q MLS revealed lower values for the hardness and Young's modulus compared to the monolithic references, not only did the TMV-Cys MLS maintain hardness and Young's modulus compared to the reference, but the mechanical performance was also even better. Overall it could be shown that an interplay between the nanocrystalline zinc oxide on one side and the organic template (both SAMs and TMV variants) on the other exists, which influences the electronic and mechanical properties of the corresponding hybrid materials. Consequently, the design of the properties of multifunctional materials by the introduction of defined and also genetically modified organic/inorganic interfaces is clearly evident.

\section{Acknowledgements}

The financial support through the priority program SPP 1569 of the Deutsche Forschungsgemeinschaft (BI 469/19-3, SCHN 375/27-3, EI 901/1-3) is gratefully acknowledged. The authors thank Marion Hagel of Nanostructuring Lab of Max Planck Institute (MPI) for Solid State Research, Stuttgart, for performing the photolithography and the Stuttgart Center for Electron Microscopy group in the same institute for providing access to the SEM facility; Maritta Dudek of MPI for Intelligent Systems for XRD measurements; and Prof. Dr Andreas Leineweber of Technische Universität Freiberg for helping with the fitting and evaluation of the data.

\section{REFERENCES}

1. Wegst UGK, Bai H, Saiz E, Tomsia AP and Ritchie RO (2015) Bioinspired structural materials. Nature Materials 14(1): 23-36.

2. Nguyen TD, Mao S, Yeh YW, Purohit PK and McAlpine MC (2013) Nanoscale flexoelectricity. Advanced Materials 25(7): 946-974.

3. Fan TX, Chow SK and Di Z (2009) Biomorphic mineralization: from biology to materials. Progress in Materials Science 54(5): 542-659.

4. Lee BY, Zhang JX, Zueger C et al. (2012) Virus-based piezoelectric energy generation. Nature Nanotechnology 7(6): 351-356.

5. Lipowsky P, Hedin N, Bill J et al. (2008) Controlling the assembly of nanocrystalline $\mathrm{ZnO}$ films by a transient amorphous phase in solution. Journal of Physical Chemistry C 112(14): 5373-5383.

6. Atanasova P, Rothenstein D, Schneider JJ et al. (2011) Virustemplated synthesis of $\mathrm{ZnO}$ nanostructures and formation of fieldeffect transistors. Advanced Materials 23(42): 4918-4922.

7. Eiben S (2018) RNA-directed assembly of tobacco mosaic virus (TMV)-like carriers with tunable fractions of differently addressable coat proteins. In Virus-derived Nanoparticles for Advanced Technologies (Wege C and Lomonossoff GP (eds)). Humana Press, Heidelberg, Germany, pp. 35-50.

8. Eiben S, Stitz N, Eber F et al. (2014) Tailoring the surface properties of tobacco mosaic virions by the integration of bacterially expressed mutant coat protein. Virus Research 180: 92-96.

9. Zhou K, Eiben S and Wang QB (2016) Coassembly of tobacco mosaic virus coat proteins into nanotubes with uniform length and improved physical stability. ACS Applied Materials \& Interfaces 8(21): 13192-13196.

10. Schneider A, Eber FJ, Wenz NL et al. (2016) Dynamic DNA-controlled 'stop-and-go' assembly of well-defined protein domains on RNAscaffolded TMV-like nanotubes. Nanoscale 8(47): 19853-19866.
11. Eber FJ, Eiben S, Jeske $\mathrm{H}$ and Wege C (2015) RNA-controlled assembly of tobacco mosaic virus-derived complex structures: from nanoboomerangs to tetrapods. Nanoscale 7(1): 344-355.

12. Chen $Q$ and Lai H (2013) Plant-derived virus-like particles as vaccines. Human Vaccines \& Immunotherapeutics 9(1): 26-49.

13. Calo A, Eiben S, Okuda M and Bittner AM (2016) Nanoscale device architectures derived from biological assemblies: the case of tobacco mosaic virus and (apo)ferritin. Japanese Journal of Applied Physics 55(3): 03DA01.

14. Koch C, Eber FJ, Azucena C et al. (2016) Novel roles for well-known players: from tobacco mosaic virus pests to enzymatically active assemblies. Beilstein Journal of Nanotechnology 7: 613-629.

15. Southan A, Lang T, Schweikert M et al. (2018) Covalent incorporation of tobacco mosaic virus increases the stiffness of poly (ethylene glycol) diacrylate hydrogels. RSC Advances 8(9): 4686-4694.

16. Pokorski JK and Steinmetz NF (2011) The art of engineering viral nanoparticles. Molecular Pharmaceutics 8(1): 29-43.

17. Fan XZ, Pomerantseva E, Gnerlich M et al. (2013) Tobacco mosaic virus: a biological building block for micro/nano/bio systems. Journal of Vacuum Science \& Technology A 31(5), https://doi.org/10.1116/ 1111.4816584.

18. Geiger FC, Eber FJ, Eiben S et al. (2013) TMV nanorods with programmed longitudinal domains of differently addressable coat proteins. Nanoscale 5(9): 3808-3816.

19. Kadri A, Maiss E, Amsharov $\mathrm{N}$ et al. (2011) Engineered tobacco mosaic virus mutants with distinct physical characteristics in planta and enhanced metallization properties. Virus Research 157(1): 35-46.

20. Atanasova P, Stitz N, Sanctis S et al. (2015) Genetically improved monolayer-forming tobacco mosaic viruses to generate nanostructured semiconducting bio/inorganic hybrids. Langmuir 31(13): 3897-3903.

21. Atanasova P (2018) Semiconducting hybrid layer fabrication scaffolded by virus shells. In Virus-derived Nanoparticles for Advanced Technologies (Wege C and Lomonossoff GP (eds)). Humana Press, Heidelberg, Germany, pp. 393-403.

22. Sanctis S, Hoffmann RC, Eiben S and Schneider JJ (2015) Microwave assisted synthesis and characterisation of a zinc oxide/tobacco mosaic virus hybrid material: an active hybrid semiconductor in a field-effect transistor device. Beilstein Journal of Nanotechnology 6: 785-791.

23. Stitz N, Eiben S, Atanasova P et al. (2016) Piezoelectric templates new views on biomineralization and biomimetics. Scientific Reports $\mathbf{6}$ : 26518.

24. Atanasova P, Kim I, Chen B, Eiben S and Bill J (2017) Controllable virus-directed synthesis of nanostructured hybrids induced by organic/ inorganic interactions. Advanced Biosystems: 1700106.

25. Lipowsky P, Hoffmann RC, Welzel U, Bill J and Aldinger F (2007) Site-selective deposition of nanostructured $\mathrm{ZnO}$ thin films from solutions containing polyvinylpyrrolidone. Advanced Functional Materials 17(13): 2151-2159.

26. Meulenkamp EA (1999) Electron transport in nanoparticulate $\mathrm{ZnO}$ films. Journal of Physical Chemistry B 103(37): 7831-7838.

27. Oh BY, Jeong MC, Ham MH and Myoung JM (2007) Effects of the channel thickness on the structural and electrical characteristics of room-temperature fabricated $\mathrm{ZnO}$ thin-film transistors. Semiconductor Science and Technology 22(6): 608-612.

28. Okamura K, Mechau N, Nikolova D and Hahn H (2008) Influence of interface roughness on the performance of nanoparticulate zinc oxide field-effect transistors. Applied Physics Letters 93(8): 083105, https:// doi.org/10.1063/1061.2972121.

29. Chung JH, Lee JY, Kim HS, Jang NW and Kim JH (2008) Effect of thickness of $\mathrm{ZnO}$ active layer on ZnO-TFT's characteristics. Thin Solid Films 516(16): 5597-5601.

30. Fanni L, Aebersold BA, Alexander DTL et al. (2014) c-Texture versus a-texture low pressure metalorganic chemical vapor deposition $\mathrm{ZnO}$ films: lower resistivity despite smaller grain size. Thin Solid Films 565: 1-6. 
Bioinspired, Biomimetic and Nanobiomaterials Volume 8 Issue BBN1
Engineered nanostructured virus/ZnO

hybrid materials with dedicated

functional properties

Atanasova, Hoffmann, Stitz et al.
31. Sun B and Sirringhaus H (2005) Solution-processed zinc oxide fieldeffect transistors based on self-assembly of colloidal nanorods. Nano Letters 5(12): 2408-2413.

32. Sun BQ, Peterson RL, Sirringhaus $H$ and Mori K (2007) Lowtemperature sintering of in-plane self-assembled $\mathrm{ZnO}$ nanorods for solution-processed high-performance thin film transistors. Journal of Physical Chemistry C 111(51): 18831-18835.

33. Hoffmann RC, Sanctis S, Erdem E, Weber S and Schneider JJ (2016) Zinc diketonates as single source precursors for $\mathrm{ZnO}$ nanoparticles: microwave-assisted synthesis, electrophoretic deposition and fieldeffect transistor device properties. Journal of Materials Chemistry C 4(30): 7345-7352.

34. Hoffmann RC, Sanctis S and Schneider JJ (2017) Molecular precursors for $\mathrm{ZnO}$ nanoparticles: field-assisted synthesis, electrophoretic deposition, and field-effect transistor device performance. Inorganic Chemistry 56(13): 7550-7557.

35. Wise SW (1970) Microarchitecture and deposition of gastropod nacre. Science 167(3924): 1486-1488.

36. Addadi L and Weiner S (1992) Control and design principles in biological mineralization. Angewandte Chemie International Edition 31(2): 153-169.

37. Sarikaya M (1994) An introduction to biomimetics - a structural viewpoint. Microscopy Research and Technique 27(5): 360-375.

38. Mayer G (2005) Rigid biological systems as models for synthetic composites. Science 310(5751): 1144-1147.

39. Calvert $\mathrm{P}$, Cesarano J, Chandra H et al. (2002) Toughness in synthetic and biological multilayered systems. Philosophical Transactions of the Royal Society A: Mathematical, Physical and Engineering Sciences 360(1791): 199-209.

40. Burghard Z, Tucic A, Jeurgens LRH et al. (2007) Nanomechanical properties of bioinspired organic-inorganic composite films. Advanced Materials 19(7): 970-974.

41. Burghard Z, Zini L, Srot V et al. (2009) Toughening through natureadapted nanoscale design. Nano Letters 9(12): 4103-4108.

42. Munch E, Launey ME, Alsem DH et al. (2008) Tough, bio-inspired hybrid materials. Science 322(5907): 1516-1520.

43. Lipowsky P, Burghard Z, Jeurgens LPH, Bill J and Aldinger F (2007) Laminates of zinc oxide and poly(amino acid) layers with enhanced mechanical performance. Nanotechnology 18(34): 345707.

44. Xu H, Xie L, Chen JB et al. (2014) Strong and tough micro/ nanostructured poly(lactic acid) by mimicking the multifunctional hierarchy of shell. Materials Horizons 1(5): 546-552.
45. Kilper S, Facey SF, Burghard Z et al. (2018) Macroscopic properties of biomimetic ceramics are governed by the molecular recognition at the bioorganic-inorganic interface. Advanced Functional Materials 28(10): 1705842

46. Zlotnikov I, Gotman I, Burghard Z, Bill J and Gutmanas EY (2010) Synthesis and mechanical behavior of bioinspired $\mathrm{ZrO}_{2}$-organic nacre-like laminar nanocomposites. Colloids and Surfaces A: Physicochemical and Engineering Aspects 361(1-3): 138-142.

47. Siglreitmeier M, Wu BH, Kollmann T et al. (2015) Multifunctional layered magnetic composites. Beilstein Journal of Nanotechnology $\mathbf{6}$ : 134-148.

48. Passerini A, Punta M, Ceroni A, Rost B and Frasconi P (2006) Identifying cysteines and histidines in transition-metal-binding sites using support vector machines and neural networks. Proteins 65(2): 305-316.

49. Rothenstein D, Claasen B, Omiecienski B, Lammel P and Bill J (2012) Isolation of $\mathrm{ZnO}$-binding 12-mer peptides and determination of their binding epitopes by NMR spectroscopy. Journal of the American Chemical Society 134(30): 12547-12556.

50. Koehler JS (1970) Attempt to design a strong solid. Physical Review B 2(2): 547-551.

51. FratzI P, Gupta HS, Fischer FD and Kolednik O (2007) Hindered crack propagation in materials with periodically varying Young's modulus - lessons from biological materials. Advanced Materials 19(18): 2657-2661.

52. Sellinger A, Weiss PM, Nguyen A et al. (1998) Continuous selfassembly of organic-inorganic nanocomposite coatings that mimic nacre. Nature 394(6690): 256-260.

53. Xia ZH, Curtin WA and Sheldon BW (2004) A new method to evaluate the fracture toughness of thin films. Acta Materialia 52(12): 3507-3517.

54. Janssen D, De Palma R, Verlaak S, Heremans P and Dehaen W (2006) Static solvent contact angle measurements, surface free energy and wettability determination of various self-assembled monolayers on silicon dioxide. Thin Solid Films 515(4): 1433-1438

55. Oliver WC and Pharr GM (1992) An improved technique for determining hardness and elastic-modulus using load and displacement sensing indentation experiments. Journal of Materials Research 7(6): 1564-1583.

56. Wang J, Li WZ, Li HD, Shi B and Luo JB (2000) Nanoindentation study on the mechanical properties of Tic/Mo multilayers. Thin Solid Films 366(1-2): 117-120.

\section{How can you contribute?}

To discuss this paper, please submit up to 500 words to the journal office at journals@ice.org.uk. Your contribution will be forwarded to the author(s) for a reply and, if considered appropriate by the editor-in-chief, it will be published as a discussion in a future issue of the journal.

ICE Science journals rely entirely on contributions from the field of materials science and engineering. Information about how to submit your paper online is available at www.icevirtuallibrary.com/page/authors, where you will also find detailed author guidelines. 